that a "plane geometry" subject to a weaker set of axioms on "points" and "lines" permits a point coordinatization $P=(x, y)$ with $x, y$ in a ring $R$ with unit, without zero-divisors, and with every two elements $(a, b) \neq(0,0)$ having a g.c.r.d. $=d$ ( $d$ right divisor of $a, b$ divisible by every right divisor of $a, b)$; set of all points on a line: $(x+a k, y+b k), x y$ fixed; $a, b$ fixed, not 0,0 , with g.c.r.d. $1 ; k$ arbitrary in $R$. The intuitive ideas in the "geometry" of the lattice points (integer coordinates) of the affine real plane are thus abstracted in equivalent geometric and algebraic forms. (Received May 20, 1942.)

\title{
Statistics and Probability
}

\section{Henry Scheffé: On the theory of testing composite hypotheses with one constraint.}

Suppose that one has a sample from a population whose distribution function depends on $l$ parameters $\theta_{1}, \theta_{2}, \cdots, \theta_{l}$, and that on the evidence of the sample it is wished to test the hypothesis $H_{0}: \theta_{1}=\theta_{1}^{\circ}$. Not only are many statistical problems of practical interest precisely of this type, but the theory of testing such hypotheses with one constraint is intimately related to Neyman's theory of confidence intervals (Proceedings of the Royal Society, 1937). Type B regions for testing $H_{0}$ were defined by Neyman (Bulletin Société Mathématique de France, vol. 64, (1935)) who gave a method of finding such regions in the case $l=2$. His theorem is generalized to the case $l \geqq 2$ in the present paper. Type $B_{1}$ regions are defined as the natural generalization of the type $A_{1}$ regions of Neyman and Pearson (Statistical Research Memoirs, 1936) to permit the presence of the nuisance parameters $\theta_{2}, \cdots, \theta_{l}$ in the distribution. Sufficient conditions are given that a type $B$ region be also of type $B_{1}$. The paper will appear in the Annals of Mathematical Statistics. (Received May 29, 1942.)

\section{TOPOLOGY}

\section{W. H. Gottschalk: On k-to-1 mappings.}

Let $A$ and $B$ be compact metric spaces and let the mapping $T(A)=B$ be continuous and at most $k$-to-1, where $k$ is an integer. Let $o(x)$ denote the Urysohn-Menger order of the point $x$. It is shown that if $y \in B$ has exactly $n$ inverse points $x_{1}, \cdots, x_{n}$, then $\sum_{i=1}^{n} o\left(x_{i}\right) \leqq k \cdot o(y)$. Suppose $A$ and $B$ are continua and $T$ is (exactly) $k$-to-1. It follows from this inequality that if $y$ is an end point of $B$, then each inverse point of $y$ is an end point of $A$. Let $P$ denote the property of being a subcontinuum of $A$ on which $T$ is $k$-to-1. It is proved that if $A$ has (the inducible property) $P$ irreducibly, then $B$ contains no end point; and, in case $k=2, B$ has the stronger property of containing no cut point. It follows that no dendrite is a continuous $k$-to-1, $k>1$, image of a continuum; or, equivalently, every continuous $k$-to-1, $k>1$, image of a continuum contains a non-cut non-end point. (Received April 11, 1942.)

\section{P. R. Halmos and Hans Samelson: On monothetic groups.}

A topological group is monothetic (van Dantzig) if it contains an element (called a generator) whose powers are dense in the group. (I) A compact abelian group is monothetic if and only if its character group is algebraically isomorphic to a subgroup of the circle. (II) A compact separable abelian group is monothetic if and only if the quotient group with respect to the component of the identity is such. (Here separability means the existence of a countable dense set.) In particular a compact separable and connected abelian group is always monothetic. (III) Almost every element (Haar 